\title{
Protée
}

\section{Les deux mémoires de Pierre Perrault}

\section{Michèle Garneau}

Volume 32, numéro 1, printemps 2004

Mémoire et médiations

URI : https://id.erudit.org/iderudit/011022ar

DOI : https://doi.org/10.7202/011022ar

Aller au sommaire du numéro

Éditeur(s)

Département des arts et lettres - Université du Québec à Chicoutimi

ISSN

0300-3523 (imprimé)

1708-2307 (numérique)

Découvrir la revue

Citer cet article

Garneau, M. (2004). Les deux mémoires de Pierre Perrault. Protée, 32(1), 23-30. https://doi.org/10.7202/011022ar

\section{Résumé de l'article}

Ce texte se penche sur l'importance de la médiation audiovisuelle dans la vision du monde de Pierre Perrault en s'appuyant à la fois sur sa pratique de cinéaste et sur celle d'écrivain de cinéma. Cette médiation audiovisuelle, dont les deux moments fondamentaux sont le tournage et le montage, et auxquels correspondent deux espèces de mémoire, aura permis au cinéaste de mettre au point une véritable méthode de perception de la réalité. L'idée centrale est que ni la mémoire électronique, recueillie au moment du tournage, ni le mémorable, recherché et construit au moment du montage, ne sont, chez Perrault, connus à l'avance. 


\section{LES DEUX MÉMOIRES DE PIERRE PERRAULT}

MiChÈLE GARNEAu

Dans son dernier grand texte sur le cinéma, L'Oumigmatique ou l'Objectif documentaire (1995), Pierre Perrault fait l'éloge de son instrument, la caméra. Davantage peut-être que dans les textes antérieurs, Perrault se confronte à la réalité donnée par l'appareil de vision, à ce qu'il appelle le "grand jeu des lentilles» (1995: 170). J'y discerne, toutefois, comme un écho au premier texte de l'auteur sur le cinéma, Discours sur la parole, écrit trente ans plus tôt ${ }^{1}$. Dans ce texte, Perrault «se prenait pour Cartier»-le titre complet est Discours sur la parole ou comment, me prenant pour Cartier, j'ai fait la découvrance de rivages et d'hommes que j'ai nommés pays et faisait l'éloge du magnétophone, de l'écoute et de la voix. Dans L'Oumigmatique, le cinéaste, qui «n'est pas Galilée», fait néanmoins l'éloge de ce qui le rapproche de ce dernier, à savoir la vision optique. "Que je ne suis pas Galilée. Pourtant je fais confiance au regard de mes objectifs. À l'impartialité des lentilles. Une simple lunette n'a-t-elle pas un jour remis à sa place la terre qui se prenait pour le nombril du monde?» (1995: 173). «L'objectif documentaire» ne serait donc pas seulement à entendre dans le sens de «but» ou encore de «résultat» que se proposerait d'atteindre le cinéaste en faisant des films documentaires, mais dans le sens de l'objectif de la caméra. En commençant son ouvrage par «La lunette de Galilée», Perrault salue l'objectivité de l'objectif, ce regard de pure visibilité qu'est le regard froid de la caméra. «J'accrédite donc le regard, impassible comme la glace, des objectifs documentaires» (1995: 17). Ne commettons pas l'erreur, toutefois, de confondre l'objectivité de la reproduction avec celle de la représentation de l'objet. L'objectivité automatique est non objectivante; elle libère l'objet du monde des représentations. Perrault n'a pas cessé de revenir sur cette question à travers un rejet de ce qu'il appelait indifféremment - ce qui aura généré beaucoup de polémiques et de confusions! - les "Écritures», "l'imaginaire», la «fiction», autant de «médiations falsificatrices» en regard de la seule qu'il légitimera afin de s'approcher du «vécu des vivants»: la médiation cinématographique.

«L'objectif documentaire», qui n'est pas l'objectivité documentaire, mais l'objectivité du regard-caméra, regard impassible comme la glace, c'est une éthique documentaire au plus près du "grand jeu des lentilles». Et il y aurait à lire, dans ce titre, ce qui constitue l'un des grands principes du cinéma, à savoir une pensée 
consciente (l'objectif du cinéaste) et une perception sans conscience (l'objectif de la caméra). La vision du monde de Perrault est inséparable de cette visibilité technique, qui est d'abord et avant tout celle d'une pénétration intensive du réel par les appareils. Or, cette pénétration ne s'effectue pas seulement au moment du tournage, par l'enregistrement automatique, mais aussi au moment du montage, par la recherche d'une logique poétique. C'est une intensification en deux temps.

J'ai parlé d'éthique. Elle concerne au plus près la méthode de perception qu'est le cinéma pour Pierre Perrault. Elle est celle de la conduite à adopter au moment de l'enregistrement, c'est-à-dire au moment du tournage, et ensuite à l'égard de ce qui a été enregistré, c'est-à-dire au moment du montage. Ces deux moments de la méthode correspondent à deux types de mémoires: d'une part, ce que Perrault luimême nomme la «mémoire électronique» ou encore la «mémoire infaillible» de l'enregistrement automatique; d'autre part, ce que j'appellerai le «mémorable ». Ce sont deux moments dans la rencontre du cinéaste avec la réalité, «une rencontre qui est loin d'aller de soi» (1995: 24). Or, si la rencontre avec la réalité est loin d'aller de soi, c'est que ni la mémoire électronique ni le mémorable ne sont, chez Perrault, connus à l'avance. La mémoire est pour lui de l'ordre de la quête, de la rencontre et de l'inspiration. C'est la recherche de quelque chose qui n'existe pas encore mais qui se pressent, qui émerge d'une réalité qui n'est pas donnée d'avance mais qui se crée sous nos yeux.

\section{LE POUVOIR RÉVÉLATEUR}

DES INSTRUMENTS NOUVEAUX

Jusqu'à la fin, Perrault s'est émerveillé du pouvoir de son instrument. Encore, en 1995, il écrivait: «Mais il faudrait finir par se rendre compte peut-être du regard neuf que la caméra nous procure» (1995: 176). Quelle est donc cette nouveauté? Je dirais que c'est l'image objective du réel. Pour la première fois, une image du monde extérieur se forme automatiquement sans intervention "artistique» de l'homme. On verra plus loin comment Perrault refuse la médiation du
Dieu-auteur, celle du texte, et, plus généralement, la médiation artistique. Si l'outil qui est le sien est fabuleux, c'est parce que, "pour la première fois dans l'histoire de l'humanité», la caméra permet à l'homme «de prolonger sa mémoire, de regarder le monde, de raconter sa propre humanité, sans avoir à invoquer les dieux de l'imaginaire» (1995: 176). Ce que Marcel L'Herbier entendait par «intelligence du cinématographe», Robert Bresson par «l'indifférence scrupuleuse d'une machine» ou encore Pier Paolo Pasolini par «la mémoire reproductive sans interprétation", c'est la réalité objective qui, pour être objective, n'est pas pour autant figée.

Voici ce que l'expérience du magnétophone et l'audition rétrospective qu'elle permet révéleront au futur cinéaste.

Petit à petit, le magnétophone m'a investi de ces pouvoirs. Et enseigné ses usages. Il m'a apprivoisé. En vérité, on ne comprend le phénomène qu'au moment où on relit la bande sonore. La parole soudain se condense. Elle est comme éclairée de l'intérieur. Et cette étrange sensation de pouvoir réentendre sans avoir à faire répéter. Et aussi la possibilité d'isoler, de rapprocher, de comparer. J'ai parfois la sensation d'entendre pour la première fois. (Perrault, 1985: 36)

Si l'homme doit se laisser apprivoiser par la machine, et non l'inverse, c'est bien parce qu'elle recèle une part «révélante», une part secrète, magique, presque mystique.

Celui qui n'a pas, une fois, capturé le langage, retenu prisonnière la parole vécue, vivante, ardente, pour la contempler à son aise, ne soupçonne pas la révélation qu'il pourrait en tirer. (Ibid.: 30)

Phénomène de double capture entre l'homme et la technique, puisque la parole capturée capture à son tour - dans le sens, cette fois-ci, de captiver celui qui l'écoute.

En vérité j'ai découvert la perspicacité d'une caméra appliquée au réel. [...] Deux yeux, deux oreilles et la fragile faculté qui oublie qui se nomme tout de même la mémoire, la mémoire humaine, n'arrivent pas à percevoir certaines choses, certaines valeurs. Marie Tremblay... la discrète Marie, je n'ai perçu son charme qu'en réecoutant mes enregistrements.

(Perrault, 1983: 45) 
Mais quelle est au juste la nature de cette puissance des instruments nouveaux? Perrault répond par une tautologie apparente, qui est un des traits rhétoriques de l'essayiste. "Que faire de ce nouveau regard sinon regarder, de cette mémoire magnifiée, sinon mémoriser?» (1992: 8). Regarder, mémoriser, mais non pas imaginer. «'ai essayé, écrit Perrault, de ne pas imaginer le paysan mais de le regarder et de l'écouter" (Écritures, 1983: 50). Les instruments ne sont pas seulement fabuleux par la perception nouvelle qu'ils nous donnent du monde, ils sont précieux dans la nécessité d'échapper à l'«image», entendue ici péjorativement au sens platonicien. Les instruments nouveaux donnent à voir et à entendre; c'est une donation. "Le don de prêter l'oreille se perd", écrit W. Benjamin (1992: 212). Chez Perrault, l'expérience du magnétophone est ce don. Une donation en même temps qu'une constitution du regard et de l'écoute, une constitution de la mémoire. Le cinéma donne à voir et à entendre. Selon Comolli:

[il] ressuscite, il «sauve» par le regard; "Sauver» signifie ici faire exister dans un film. [...] La chose "sauvée» par le film peut très bien être perdue dans le monde, peu importe, elle reste à advenir dans le film à chacune de ses projections. (1994: 50)

Après l'expérience du magnétophone, Perrault fait celle de la caméra synchrone ${ }^{2}$, et c'est en ce sens qu'il parlera de «mémoire décuplée»: «Dans la mesure où une caméra pouvait, sur le champ, capter, en synchro, l'image et le son, nous nous sentions responsables de cette mémoire décuplée» (1992: 8). Respect, privilège et engagement sont les termes par lesquels Perrault aborde la mémoire électronique et c'est à partir d'elle que le mémorable se construira.

\section{SAVOIR OU VÉRITÉ DE LA MÉMOIRE}

La caméra du «direct», chez Perrault, n'est pas un outil d'information pour délivrer un savoir, mais pour découvrir et s'approcher d'une vérité. Cette vérité ne sera pas tributaire de la captation d'une réalité préexistante, mais de ce que la caméra parviendra à stimuler. C'est la méthode d'un tournage, doublée de celle d'un montage, qui fera advenir - ou pas - la vérité. Invention toujours périlleuse et fragile des scènes du filmage. En d'autres termes: la vérité d'une situation n'est jamais donnée d'avance, elle est de l'ordre de l'événement.

Parlant de La Bête lumineuse, Perrault a écrit: «Il s'est institué en événement. Peut-être en tragédie» (1983: 115). J'oppose ici, dans la captation de la mémoire électronique comme dans celle de la fabrication du mémorable, savoir et vérité, l'assurance des règles et des résultats du savoir, à l'aléa des coups de la vérité. On peut échouer dans la quête d'une vérité - vérité qui, chez Perrault, est celle de la rencontre-, mais pas dans la diffusion d'un savoir. «La vérité, nous dit Perrault, n'est pas une dimension particulière à une forme de cinéma: elle est plutôt une qualité de l'inspiration" (ibid.: 13). L'inspiration atteste qu'une rencontre a bel et bien eu lieu.

Cette conception du cinéma me semble en opposition radicale avec celle qui demande au documentaire de livrer une bonne information. «L'information, écrit W. Benjamin, est incompatible avec l'esprit même de la narration. Le narrateur né, ajoute-t-il, débarrasse toujours une histoire, lorsqu'il la raconte, de toute explication" (1992: 209).

Distinguons donc l'acte qui consiste à livrer une information de celui qui consiste à narrer un vécu ou une expérience. Pour Benjamin, l'art de raconter est l'art d'échanger des expériences; par expériences, l'auteur entend non l'observation scientifique mais l'exercice populaire de la sagesse pratique. C'est un art - ou encore une faculté - qui toucherait à sa fin, parce que «le cours de l'expérience a baissé». L'art de narrer est en déclin parce que l'aspect épique de la vérité, la sagesse, tend à disparaître.

Mais ce soir-là, parmi les horloges affectées à énumérer la nuit et à recommencer le jour, grâce au magnétophone, en toute innocence, j'imprimais cette lourde sagesse. Et je lui cherchais un sens. Bien sûr, elle ne parlait qu'au passé. Le récit, que peut-il sinon hommager le passé... le vécu... sinon s'approfondir sur ce qui est ...sur ce qui a été? Et les récitants récitent leur vie.

L'art de conter se ferait de plus en rare, selon W. Benjamin, car une place de plus en plus grande est donnée à l'information. Les événements de notre temps profitent à l'information et presque plus rien 
ne profite à la narration $(1992: 211)$. Or, toute l'entreprise de Perrault est de trouver ou encore de susciter des situations qui profitent à la narration. La suite des propos de Benjamin, qui met en évidence les liens du travail (artisanal) et de la narration, nous permet de comprendre pourquoi Perrault n'a filmé que les artisans et rarement les ouvriers, reproche qui lui fut souvent fait. C'est que l'artisan, contrairement au travailleur d'usine, peut raconter en même temps qu'il travaille. "Le don de prêter l'oreille se perd, écrit Benjamin. Il se perd parce qu'on n'écoute plus en tissant, en filant» (1992: 212). Dans tous les films de Perrault, les gens se racontent en travaillant. Filmer le travail, filmer les hommes pendant qu'ils travaillent (la construction de l'embarcation dans Les Voitures d'eau est exemplaire à cet égard). On a souvent reproché à Perrault de faire l'éloge de la technologie du passé, de l'artisanat et des techniques préindustrielles. Or, si Perrault s'y attarde, c'est pour des raisons essentiellement - ou presque!narratives.

\section{UNE MÉTHODE DE PERCEPTION}

Voyons ce qu'il en est plus précisément de ces deux opérations dans la méthode du cinéaste. Je dirais que le moment du tournage correspond, chez Perrault, à un défrichage, tandis que le montage pourrait être apparenté à une opération de déchiffrement. Le tournage, c'est l'événement de parole, le trajet selon lequel des êtres parlants se vouent à la vérité de leur parole.

J'essaie de découvrir en chaque homme une inspiration, écrit le cinéaste. Je l'attends. Quand elle arrive je la filme, et j'efface ensuite ce qu'il y a avant. C'est du griffonnage. Comment une personne peut devenir, à l'intérieur de son discours, lumineuse. ${ }^{3}$

Le montage sera la réinscription de cet événement dans l'équivalence d'un récit, d'une logique poétique. Le tournage, telle une lecture, et le montage, telle une écriture. Mais la lecture du tournage ne se consacre pas seulement à la parole. Perrault trouve de la parole partout. Ce cinéma de la parole n'est pas seulement celui, comme on l'a souvent dit, de la parole des gens. Il s'agit aussi de faire parler ce qui est muet. Toute une mémoire est tapie dans le matériau, ce «témoin muet». La théorie du témoin muet, écrit Jacques Rancière, et qu'il prélève à partir de l'écriture michelétiste de l'histoire, valorise un discours du lieu et des choses, un discours de ce qui n'a pas coutume de parler (1992: 115). Le cinéma ne serait-il pas l'art qui peut le mieux «énumérer tous les signes muets par lesquels l'homme s'est dit" (ibid.: 119) ou encore "faire entendre le sens à l'état d'en-fance, le sens non bavard, inscrit dans la texture des choses" (ibid.: 124)? Il peut, par exemple, et mieux que la littérature, inscrire une parole dans la ressemblance à soi d'un paysage ${ }^{4}$. Le tournage sera donc aussi une lecture des signes muets écrits sur la configuration d'un lieu, d'un paysage, d'une maison, d'un visage, d'une main. Il fera voir et entendre "ce grand poème hiéroglyphique inscrit sur la chair même des choses" (Rancière, 1998: 174).

«Tourner un film pour nous, c'est chercher dans le présent une réalité dispersée que nous prétendons rapailler par le montage ${ }^{5}$. Mais « rencontrer la réalité », quand l'enregistrement ne relève pas de choses déjà gagnées, déjà dominantes, déjà majoritaires, ne va pas de soi. "Mais il me semble bien, écrit Perrault, qu'on ne reconnaisse que l'exprimé, le cinématographié, le possédé» (1983: 44). Peut-être pourrions-nous ajouter: le mémorisé. Il faut être aux aguets:

[...] le cinéma que je fais est un cinéma qui cherche à être aux aguets et qui, évidemment, à un moment donné, est obligé de prendre une piste, une idée [...], une petite hypothèse ridicule puis, tout à coup, il y a le hasard et puis tu suis le hasard [...], j'ai toujours l'impression quand je fais un film que je suis à la chasse [...], il y a des pistes et je me laisse inspirer par des pistes. Au bout, il y a une bête ou il n'y en a pas. ${ }^{6}$

Rien ne préexiste au tournage, sinon une "petite hypothèse": on accumule sans programme fixe, on impressionne une très grande quantité de pellicule dont la destination n'est ni déterminée ni connue. La mémoire électronique se constitue sous nos yeux, par le tracé de la caméra, une caméra qui cherche à se laisser toucher par les traces, celles de la vie, d'un vivre-ensemble. Ce que la caméra enregistreuse va faire sortir de l'ombre et rencontrer ne se dévoile parfois 
qu'au moment du montage. Sur le tournage abitibien, qui a duré plusieurs années, Perrault a déclaré en entrevue:

Et c'est au montage que nous avons découvert les films. Que les

films se sont démarqués. Comme d'eux-mêmes. En observant une

logique à laquelle nous devions obéir. Dans un ordre qui n'avait

rien à voir avec la chronologie du tournage. Nous avons donc

fait ces films les uns après les autres. En épuisant les logiques.

(Séquences, 1981: 16)7

Épuiser les logiques, c'est en élire une. Le montage est primordial: il faut trouver, voire découvrir la logique du film. C'est un «direct» qui reconstruit la réalité saisie au tournage par le montage. Un tel cinéma de l'événement n'est possible qu'à la condition de concevoir le travail de réalisation comme la recherche d'une vérité qu'il n'est possible de déterminer qu'a posteriori, comme accouchée par l'acte de l'écriture et par lui seul. Or, le plus souvent dans le documentaire «traditionnel», on a affaire à un travail qui s'apparente à un processus de représentation (au sens de la reproduction de ce qui existe déjà). Ces documents servent un savoir que le cinéma ne peut que reproduire, un savoir antérieur au moment du tournage. Jean-Louis Comolli parle «d'antériorité de la réalité filmée et d'extériorité du filmage» (1969: 50). «C'est le montage parfois qui trouve, nous dit Perrault. Le tournage fouille, glane, sans trop savoir où il va» ${ }^{8}$. Il faut énormément de matériel (pellicule) pour créer, trouver le récit et établir une continuité. «Mais où se trouve ou plutôt comment découvre-t-on la logique d'un film? À tâtons. Petit à petit. En comparant. En éliminant. En poursuivant la bête lumineuse» (Séquences, 1981: 42). Pas plus que le tournage, le montage n'est illustratif. Il n'y a pas d'emblée une réalité mémorable qu'on s'empressera d'enregistrer et de monter afin de la révéler au spectateur. La révélation est inséparable des pouvoirs propres aux instruments - caméra filmante, table de montage-, de ce qu'ils parviendront à révéler.

Perrault, qui ne croit pas à l'absolu du montage dans la re(création) de la réalité, prétendra toujours qu'il n'y a qu'un seul montage possible à ses yeux. Une réalité a été captée qu'il faut à la fois respecter et déchiffrer. En d'autres termes: il y a un réel à cette réalité - le mémorable - qui finira bien, si on sait la lire, par apparaître. Le montage en rendra seulement le surgissement plus lumineux.

Ce qui donne la dimension poétique, c'est le montage. Sans comparaisons, rapprochements, contrastes, contradictions, etc., [...] les faits enregistrés restent pour ainsi dire lettre morte. Comme une eau brouillée. Autrement dit, le montage décante. Il est une façon précise de lire dans le fouillis du réel. (1971: 28)

Ce qui contribue à l'agrandissement épique dans tous les films du cycle abitibien, ce qui magnifie et intensifie la représentation, ce sont les incessants chassés-croisés de temporalités et l'enchevêtrement des récits - toujours exemplaires. C'est une mythification du vécu par redondance narrative. C'est ainsi que le mémorable se construit. C'est une composition, comparable, musicalement, au "thème et variations"; à chaque séquence, le même motif revient, avec un déplacement infime. Les films de Perrault sont un ressassement des mêmes motifs et des mêmes tableaux. Il n'y a pas de progression d'une partie à l'autre, pas d'introduction ou de conclusion ouvrant ou fermant l'exposé, mais des parties qui, régulièrement, s'interrompent, reviennent au point de départ et repartent à zéro pour examiner un autre détail et en saisir, enchâssé, le tout. Inlassablement, comme peutêtre la mémoire elle-même, la pensée recommence et revient minutieusement sur la chose même.

\section{LA MÉDIATION FALSIFICATRICE}

Ce cinéma ne s'écrit pas, répétera Perrault, il est tributaire de l'événement. Perrault n'écrit pas son film avant, dans un scénario, mais après, dans des textes qui relatent une aventure souvent éreintante, des textes qui inscrivent sa parole

[...] ailleurs, dans une démarche d'écriture qui n'a peutêtre que la valeur de me reprendre en main après chaque aventure cinématographique, dans le contenu de l'autre. (Écritures, 1983: 45)

En esquivant l'étape du scénario, le "direct» de Perrault crée une manière de faire dans laquelle l'étape du découpage fait partie intégrante du sujet. C'est sur le terrain, à même le terrain, que le sujet 
s'élabore. Le développement du sujet - trop souvent littéraire dans le cinéma documentaire comme dans celui de fiction, c'est-à-dire strictement enfermé à l'intérieur de l'étape de l'écriture scénaristique - se fait à même les surprises et les aléas du terrain.

Un film n'est jamais qu'une hypothèse. Évidemment, je ne parle pas de fiction et de scénarios. Une hypothèse qui n'est pas fictive. Bien réelle. [...] Mais plus le film est bon, plus peutêtre il se rapproche d'un vécu, d'une substance incompatible à la fiction, à l'imaginaire. (Séquences, 1981: 42)

La médiation du texte est refusée au profit de la seule médiation audiovisuelle. Ce que Jacques Derrida a écrit, à partir de l'expérience théâtrale d'Antonin Artaud, s'applique parfaitement à l'expérience cinématographique de Perrault. Derrida fait ressortir les composantes de ce qu'il appelle la "scène théologique», dont le référent ultime est le Texte. Le Texte, nous dit Derrida, est une «arme» qui permet de "surveiller" et de "commander le temps et le sens de la représentation»:

La scène est théologique tant que sa structure comporte, suivant toute la tradition, les éléments suivants: un auteur-créateur qui [...] armé d'un texte, surveille, rassemble et commande le temps ou le sens de la représentation, laissant celle-ci le représenter dans ce qu'on appelle le contenu de ses pensées, de ses intentions, de ses idées. (1967: 345-346)

Perrault fait-il des films qui reflètent ses pensées, ses intentions, ses idées? Plus d'une fois, la question a été débattue, mais le cinéaste s'est toujours défendu de s'exprimer en son propre nom. «S'il m'arrivait d'avoir à m'exprimer au lieu d'exprimer le tournage, je possède d'autres outils pour le faire» (Séquences, 1981: 18). Entre la réalité du "vécu des vivants», il n'y aura pas d'autre médiation que la médiation audiovisuelle. Car ce qu'il faut éviter prioritairement, c'est la «structure représentative» qui, comme l'écrit Derrida, garde «avec ce qu'on appelle le "réel" [...] un rapport imitatif et reproductif".

Représenter par des représentants, metteurs en scène ou acteurs, interprètes asservis qui représentent des personnages qui, d'abord par ce qu'ils disent, représentent plus ou moins la pensée $d u$ "créateur». Esclaves interprétants, exécutant fidèlement les desseins providentiels du «maître». Qui d'ailleurs - et c'est la règle ironique de la structure représentative qui organise tous ces rapports - ne crée rien, ne se donne que l'illusion de la création puisqu'il ne fait que transcrire et donner à lire un texte dont la nature est nécessairement elle-même représentative, gardant avec ce qu'on appelle le «réel» [...] un rapport imitatif et reproductif.

$(1967: 345-346)$

Ce que la pratique du «direct» viendra ébranler, c'est: [la] notion selon laquelle celui qui fait des films serait un metteur en scène, c'estàddire un monsieur qui, à partir d'un sujet, fait ou fait faire un scénario qu'ensuite il mettra en images. 9

Perrault refuse la médiation du Dieu-auteur, tout comme celle du texte. «Et si je ne voulais pas m'exprimer même par le truchement de personnages nés de mon imaginaire. Si je voulais éviter cette médiation falsificatrice» (Écritures, 1983: 50). Il sera ce "cinéaste des autres":

Non pas par humilité, mais parce que je pense que l'écriture n'est pas le service de l'écrivain. Mais celui des autres. On peut bien sûr penser autrement. C'est mon choix. Un choix politique peut-être. Historique sans doute. Un choix du Nouveau Monde aussi. Il est inutile de refaire la littérature des Puissances. Je me cherche un destin dans un certain refus des formes consacrées.

(Écritures, 1983: 48)

\section{LA MÉDIATION AUDIOVISUELLE:}

PERFORMANCE CONTRE MIMÉSIS

Jean-Louis Comolli a raison d'écrire qu'un

[...] lien nouveau et fort unit le cinéma au vécu: la vie n'est plus "représentée» par le cinéma, celui-ci n'est plus l'image - ou le modèle - de celle-là. Ensemble ils se parlent et se produisent l'un l'autre. (1969: 52)

Dans cette manière de concevoir le cinéma - au sens d'un mode d'être, au sens d'une manière de s'engendrer soi-même par la magie d'une caméra, une manière jaillissante ${ }^{10}$-, nous ne sommes pas très loin de cette idée de "performance» que P. Zumthor oppose à la «mimésis». La «performance» récuse la mimésis et choisit d'emblée le parti d'un art sevré pour nous de l'antique illusion représentative [...]. [U]n être humain a lieu, ici, devant moi, sur scène ou à l'écran.

$(1983: 286)$ 
Ce qu'il faut voir, c'est qu'il s'agit de mettre en place un dispositif qui permet d'exclure cette figure de l'extériorité qu'est l'imitation. Pour Perrault, dont les prises de position ont été plus d'une fois jugées «intempestives", le régime de l'imitation, au nom d'un cinéma du "vécu», est mis en accusation. Si Perrault cherche à effacer la répétition, c'est parce qu'elle sépare d'elle-même la force, la présence, la vie. "[I]naccessible limite d'une représentation qui ne soit pas répétition, d'une re-présentation qui soit présence pleine [...]", écrit Derrida au sujet d'Artaud (1967:

364). Nous sommes, avec Pierre Perrault, en présence d'un même extrémisme, d'une même résistance. Perrault cherche une procédure de sens radicalement autre que celle de la mimésis, une procédure antimimétique. D'où la fonction fabulatrice. Il y aurait, dans la destruction du principe de mimésis, une exigence, écrit J. Rancière: celle que «l'histoire de la vie profonde des masses succède à la chronique royale» (1992: 106). Perrault dit la même chose lorsqu'il écrit:

Chaque époque produit des images royales, des images qui normalisent. C'est l'occupation du territoire de l'âme par un pouvoir. De plus en plus puissant d'ailleurs. Électronique. [...] C'est alors que je me suis intercédé Alexis, et Grand-Louis, et Marie, etc. Pour savoir qui j'étais. En sorte que pour me dire, il me suffit de leur donner la parole. Puisqu'ils sont ma littérature.

(Écritures, 1983: 56)

C'est en se déliant de la mimésis que le cinéma pourra rendre l'histoire possible comme discours de vérité. Car la mimésis suppose toujours une extériorité: celle du modèle qu'on imite. Dans sa condamnation du régime fictionnel, Perrault est platonicien. «Mais le ciel de Platon n'est pas celui d'Homère. À chacun de choisir le ciel qui lui convient. J'ai fait mon choix» (1995: 25). Perrault est platonicien dans la mesure où sa réflexion esthétique procède d'une politique, d'un ethos de la cité. Le régime fictionnel accroît les médiations, c'est une dépossession sur le mode identificatoire, une perte de soi dans l'identification il faudrait plutôt dire: dans des identifications multiples. Qu'adviendrait-il d'une cité où tous se laisseraient contaminer par les modèles? Perrault veut libérer l'homme québécois de ses modèles, de ses idolâtries. Le proposer à lui-même. «Est-il possible d'échapper à l'entreprise idolâtre?" 11 .

Nous ne sommes pas très loin, ici, du constat célèbre de Guy Debord (1967): le statut de souveraineté absolue de l'économie marchande, qui manipule la perception collective, s'empare de la mémoire et de la communication sociale pour les transformer en une unique marchandise spectaculaire. Le spectacle, c'est le monde réel qui se transforme en images, ou les images qui deviennent réelles, qui se substituent au réel. Dans la perspective de Guy Debord, le spectacle n'est plus que la "pure forme de la séparation ". Il en va de même chez Perrault, pour qui le spectacle n'est pas simplement la sphère des images, mais un rapport social entre des personnes, médiatisé par des images. D'où l'idée d'une expropriation, d'une aliénation de la socialité. Conséquemment, cette "tentative, nous dit Perrault, de retenir le sol érodé par les slogans [...]» (1985: 174).

En allant au-devant de ce qui ne se laisse pas approcher (le bœuf musqué, dit l'oumigmag) et, plus fondamentalement, de ce qui ne parle pas, c'est la «distance infranchissable» qui semble être devenue le véritable sujet des deux derniers films de Perrault. On pourrait le dire autrement: c'est la procédure d'approche du réel que permet la médiation cinématographique qui intéresse ici Perrault, davantage peut-être que le bouf musqué. À partir de la sauvagerie irréductible de l'animal, Perrault expérimente des postures de cinéaste. Attente, guet, qui-vive, distance respectueuse, apparition, irruption, dispersion, nomination sont les éléments de ce grand poème cinématographique. Dans le livre L'Oumigmatique ou l'Objectif documentaire, on lit: Il faut tout essayer. S'approcher jusqu'à voir dans les yeux. Élargir le champ de vision pour inclure le paysage, la colline, la mer, le ciel. Mais on ne voit même plus le troupeau. Puis faire abstraction du paysage, l'exclure. S'emparer de la géographie où la bête disparaît, puis la négliger. Abstraire la bête de la vallée. L'extraire du troupeau. Ne plus voir ni neige ni herbe. Extraire la bête à son tour. Estil possible de ne voir que la tête. La distance le permet-elle? Et de la tête que la corne du regard? Et du regard que l'œil des intentions? (1995: 170) 
La concision du style, qui évoque le Beckett du Dépeupleur, donne raison à Yves Lacroix quand il écrit: «[...] qu'il faudrait bien examiner un jour les raisons de l'antagonisme hyperbolique qu'après trente ans d'écriture l'écrivain dénonce avec autant de virulence entre l'oral et l'écrit» (1999: 80). Ardent défenseur de la culture orale ${ }^{12}$, Perrault n'en est pas moins un grand écrivain. Mais ce qui le caractérise, pour ne pas dire le singularise, c'est la séparation très nette qu'il établit entre la médiation audiovisuelle, la méthode de perception qu'il en tire à partir de son objectif documentaire (dans les deux sens prélevés plus haut) et l'essai littéraire ou encore la poésie. En lui coexistent deux hommes: un homme de l'oralité et un homme de l'écriture. «À chaque époque, écrit Paul Zumthor, coexistent et collaborent des hommes de l'oralité et des hommes de l'écriture» (1983: 35). S'il faut convertir les signes morts de l'écriture en leur vérité vivante cinématographique, on peut, en retour, «se reprendre en main» après chaque aventure cinématographique. C'est le sens des essais de Perrault sur ses propres films. À quelle espèce de mémoire pourrions-nous les associer? Perrault, encore une fois, répond: "J'écris parfois pour faire le point de mes rencontres avec le vécu des vivants. Pour les retrouver" (Écritures, 1983: 45). Aller à la rencontre des hommes par la caméra et y revenir par l'écriture afin de se remémorer une expérience que seule la médiation audiovisuelle pouvait permettre.

\section{Notes}

1. Ce texte figure dans De la parole aux actes. Essais (1985: 7-39).

2. Synchronisme: enregistrement simultané de l'image et du son.

3. Propos de P. Perrault, tiré du film de J.-D. Lafond: Les Traces du rêve (1986).

4. C'est ce que j'ai tenté de montrer dans mon article: «Le paysage dans la tradition documentaire québécoise : un regard off sur la parole» (2001: 127-143).

5. Propos de P. Perrault, tiré du film de J.-D. Lafond: Les Traces du rêve (1986).

6. Ibid.

7. Amorcé en 1972 sous le titre de travail "Abitibi-Baie-James", le cycle abitibien de P. Perrault comprend 4 films: Un royaume vous attend (1975), Le Retour à la terre (1976), C'était un Québécois en Bretagne, Madame (1977) et Gens d'Abitibi (1980).
8. Propos de P. Perrault, tiré du film de J.-D. Lafond: Les Traces du rêve (1986).

9. Propos de A. S. Labarthe rapportés par N. Burch (1986: 200).

10. L'expression est de G. Agamben (1990).

11. Recherches amérindiennes, 1981: 13.

12. «Et si toute réflexion sur l'homme devait débuter par une mise en question de l'écriture, ce monarque un peu trop absolu de l'intelligence, par une remise en liberté de la parole analphabète comme source de tout langage"? ("Discours sur la parole», dans P. Perrault, 1985: 13).

\section{RÉFÉRENCES BIBLIOGRAPHIQUES}

AgAmBEN, G. [1990] : La communauté qui vient - Théorie de la singularité quelconque, Paris, Seuil, coll. "La librairie du XX $\mathrm{X}^{\mathrm{e}}$ siècle».

BENJAMIN, W. [1992]: "Le narrateur», Écrits français, Paris, Gallimard. Bresson, R. [1975]: Notes sur le cinématographe, Paris, Gallimard. Burch, N. [(1967) 1986]: Praxis du cinéma, Paris, Gallimard, coll. "folio essais".

Comolli, J.-L. [1969]: "Le détour par le direct (1)", Cahiers du cinéma, $\mathrm{n}^{\circ} 209,48-53$;

_ [1994] : «La ville filmée", Regards sur la ville, Paris, Éd. du Centre Pompidou, 13-60.

Deleuze, G. [1985] : Cinéma II - L'image-temps, Paris, Minuit. DERRIDA, J. [1967]: L'Écriture et la Différence, Paris, Seuil. Debord, G. [1967]: La Société du spectacle, Paris, Gallimard. GARNEAU, M. [2001]: «Le paysage dans la tradition documentaire québécoise : un regard off sur la parole», Cinémas ("Le paysage au cinéma»), sous la dir. d'A. Costa, automne, 127-143.

LACROIX, Y. [1999], "Suite fraternelle», dans P. Warren (sous la dir. de), Pierre Perrault, cinéaste-poète, Montréal, L'Hexagone, coll. «Itinéraires », 7693.

LAFOND, J.-D. [1988]: Les Traces de rêve, Montréal, L’Hexagone.

L'Herbier, M. [1977]: Intelligence du cinématographe, Paris, Aujourd'hui. Perrault, P. [ 1983]: Caméramages, Montréal et Paris, L'Hexagone et Edilig;

— [1985]: De la parole aux actes, essais, Montréal, L'Hexagone ; [1992]: Pour la suite du monde. Récit (photographies de M. Brault), Montréal, L'Hexagone;

[1995]: L'Oumigmatique ou l'Objectif documentaire (récit du tournage du film et essai sur le documentaire), Montréal, L'Hexagone. PASOLINI, P.P. [1976]: L'Expérience hérétique, Paris, Ramsay.

RANCIÈRE, J. [1992]: Les Mots de l'histoire - Essai de poétique du savoir, Paris, Seuil;

_ [1998]: La Parole muette. Essai sur les contradictions de la littérature, Paris, Hachette Littératures.

ZumTHOR, P. [1972]: Essai de poétique médiévale, Paris, Seuil;

_ [1983]: Introduction à la poésie orale, Paris, Seuil.

\section{Autres textes}

[1971]: Pierre Perrault, Cinéastes du Québec, vol. v, Montréal, CQDC. [1981]: Recherches amérindiennes au Québec [1981]: vol. X, no 4.

[1981]: Séquences, "Dialogue avec Pierre Perrault", Montréal, no 39, 12. 22.

[1983]: Écritures de Pierre Perrault, "Cinéma du réel et cinéma de fiction: vraie ou fausse distinction? Dialogue entre Pierre Perrault et René Allio", Actes du colloque "Gens de paroles " (Les dossiers de la cinémathèque), Montréal, Maison de la culture de La Rochelle, La cinémathèque québécoise et Edilig, vol. 11, $42-57$. 